\title{
Retinal Angiomatous Proliferation in an Eye with Cuticular Drusen
}

\author{
Atsuko Sato Nami Senda Emi Fukui Kouichi Ohta \\ Department of Ophthalmology, Matsumoto Dental University, Shiojiri, Japan
}

\section{Key Words}

Cuticular drusen - Basal laminar drusen - Retinal angiomatous proliferation · Spectral domain optical coherence tomography

\begin{abstract}
Purpose: To report the presence of retinal angiomatous proliferation (RAP) in an eye with cuticular drusen detected by fluorescein and indocyanine green angiography by confocal scanning laser ophthalmoscopy and by spectral-domain optical coherence tomography (SDOCT). Methods: Case report of a 65-year-old Japanese woman with cuticular drusen. Results: At her first ophthalmic examination, her visual acuity was 20/20 in both eyes. An ophthalmoscopy showed many small subretinal pigment epithelial deposits in both eyes. These deposits had a 'saw-tooth pattern' in the SD-OCT images. During the follow-up examination, retinal hemorrhages were observed, and fluorescein angiography showed a 'stars-in-the-sky' appearance and intraretinal neovascularization. The patient was diagnosed with cuticular drusen associated with RAP. Conclusion: We suggest that the cuticular drusen were associated with RAP, so periodic follow-up examinations are needed for patients with cuticular drusen for the early detection and treatment of RAP.

(C) 2015 S. Karger AG, Basel
\end{abstract}

\section{Introduction}

Cuticular drusen, also called basal laminar drusen, appear ophthalmoscopically as small, yellow deposits under the retinal pigment epithelium (RPE), and they have a characteristic 'stars-in-the-sky' or 'milky way' pattern in fluorescein angiograms [1, 2].

Fine details of cuticular drusen have been obtained by high-resolution, spectral-domain optical coherence tomography (SD-OCT) [3-5]. B-scan SD-OCT images show that the cuticular drusen have moderate hyperreflectivity and a 'saw-tooth pattern'.

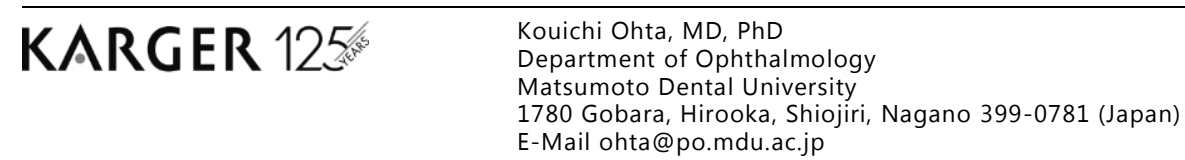


Sato et al.: Retinal Angiomatous Proliferation in an Eye with Cuticular Drusen

Cuticular drusen have also been reported in eyes with vitelliform macular detachment [6-8] and age-related macular degeneration (AMD), but the visual prognosis is better for patients with AMD. Cuticular drusen are also found in eyes with geographic atrophy. A recent study reported that choroidal neovascularization is present in up to one third of the patients with cuticular drusen [6].

The incidence of cuticular drusen is predominantly seen in Caucasian women [7]. We report a Japanese woman with cuticular drusen, and our examinations have identified a retinal angiomatous proliferation (RAP) by an instrument that allows simultaneous recordings of confocal scanning laser ophthalmoscopy (cSLO) and SD-OCT (Spectralis HRA + OCT; Heidelberg Engineering, Heidelberg, Germany).

\section{Case Report}

A 65-year-old Japanese woman complained of blurred vision in both eyes. At her first ophthalmic examination, her visual acuity was $20 / 20$ in both eyes. An ophthalmoscopy showed many small sub-RPE deposits in both eyes (fig. 1a, b). The SD-OCT images showed nodular elevations of the RPE which had a 'saw-tooth pattern' (fig. 2). This blunted triangular or prolapsed shape has been reported to be typical for cuticular drusen. Reticular pseudodrusen were also seen in both eyes. The scotopic and photopic full-field electroretinograms were reduced, and Goldmann perimetry showed a decrease in the sensitivity in the pericentral field in both eyes. We diagnosed her with cuticular drusen, and she was placed on a periodic follow-up examination schedule.

Six months after the initial examination, a fundus examination showed intraretinal hemorrhage (fig. 3a) in the left eye, and her visual acuity decreased to 20/40. Autofluorescencent images showed mottled hyper- and hypofluorescent regions (fig. 3b). Fluorescein and indocyanine green angiography by cSLO showed an early filling of an intraretinal neovascularization (fig. 3c, d) and late leakage. The fluorescein angiography images had a 'starsin-the-sky' appearance in the area of the cuticular drusen, and the SD-OCT images showed subretinal exudative changes with a 'saw-tooth pattern' (fig. 3e). RAP was then diagnosed. We recommended antivascular endothelial growth factor therapy, photodynamic therapy and/or sub-Tenon's triamcinolone injections. However, she did not accept treatment because of the potential of complications. Twelve months later, her visual acuity was 20/20 in the right eye and 20/200 in the left eye without any new retinal hemorrhages.

\section{Discussion}

Drusen vary in size, color, number and location. The soft drusen are well known to be risk factors for the development of AMD, and recently subretinal drusenoid deposits, called reticular pseudodrusen, were reported to be associated with RAP [9]. Cuticular drusen, soft drusen and reticular pseudodrusen were reported to have common components $[3,10]$, although each type of drusen has a distinct morphological appearance and location on the SD-OCT images. Ueda-Arakawa et al. [9] reported that the prevalence of reticular pseudodrusen was lower in Japanese AMD patients than in Caucasians, and they suggested that certain features of reticular pseudodrusen were ethnicity dependent. Cuticular drusen is a rare retinal disorder, and as best as we know, this is the first report of cuticular drusen in Asians. 
Sato et al.: Retinal Angiomatous Proliferation in an Eye with Cuticular Drusen

An association of acquired vitelliform lesions with cuticular drusen is known, but an association with AMD is unclear. Choroidal neovascularization has been reported to occur in $10-31 \%$ of eyes with cuticular drusen [2, 6, 7], and Guigui et al. [11] reported that photodynamic therapy was successful in treating these neovascularizations. These reports did not mention any subclassifications of AMD such as those with RAP or polypoidal choroidal vasculopathy. Performing periodic examinations by SD-OCT may possibly detect the presence of RAP in eyes with cuticular drusen more frequently. We cannot conclude, based on this case only, that cuticular drusen is a risk for RAP such as it is the case with soft drusen or reticular pseudodrusen. However, close observation is needed for patients with cuticular drusen associated with abnormal RPE.

\section{Conclusion}

We reported a case of RAP in an eye with cuticular drusen detected by SD-OCT and fluorescein and indocyanine green angiography. Cuticular drusen was diagnosed by a 'stars-inthe-sky' appearance in the fluorescein angiograms and a 'saw-tooth pattern' in the SD-OCT images. We recommend periodic follow-up examinations for patients with cuticular drusen for the early detection of RAP.

\section{Acknowledgement}

We thank Prof. Duco Hamasaki of the Bascom Palmer Eye Institute for editing the paper.

\section{References}

1 Agarwal A: Basal laminar drusen and macular degeneration; in Agarwal A (ed): Gass' Atlas of Macular Diseases, ed 5. Philadelphia, WB Saunders, 2011, pp 132-135.

-2 Boon CJ, van de Ven JP, Hoyng CB, den Hollander AI, Klevering BJ: Cuticular drusen: stars in the sky. Prog Retin Eye Res 2013;37:90-113.

-3 Spaide RF, Curcio CA: Drusen characterization with multimodal imaging. Retina 2010;30:1441-1454.

-4 Finger RP, Issa PC, Kellner U, Schmitz-Valckenberg S, Fleckenstein M, Scholl HPN, Holz FG: Spectral domain optical coherence tomography in adult-onset vitelliform macular dystrophy with cuticular drusen. Retina 2010;30:1455-1464.

-5 Leng T, Rosenfeld PJ, Gregori G, Puliafito CA, Punjabi OS: Spectral domain optical coherence tomography characteristics of cuticular drusen. Retina 2009;29:988-993.

6 Cohen SY, Meunier I, Glacet-Bernard A, Coscas GJ: Visual function and course of basal laminar drusen combined with vitelliform macular detachment. Br J Ophthalmol 1994;78:437-440.

7 Gass JD, Jallow S, Davis B: Adult vitelliform macular detachment occurring in patients with basal laminar drusen. Am J Ophthalmol 1985;99:445-459.

-8 Lima LH, Laud K, Freund KB, Yannuzzi LA: Acquired vitelliform lesion associated with large drusen. Retina 2012;32:647-651.

-9 Ueda-Arakawa N, Ooto S, Nakata I, Yamashiro K, Tsujikawa A, Oishi A, Yoshimura N: Prevalence and genomic association of reticular pseudodrusen in age-related macular degeneration. Am J Ophthalmol 2013;155:260-269.

10 Russell SR, Mullins RF, Schneider BL, Hageman GS: Location, substructure, and composition of basal laminar drusen compared with drusen associated with aging and age-related macular degeneration. Am J Ophthalmol 2000;129:205-214.

11 Guigui B, Martinet V, Leveziel N, Coscas G, Soubrane G, Souied EH: Photodynamic therapy for choroidal neovascularization secondary to basal laminar drusen. Eye 2009;23:2115-2118. 

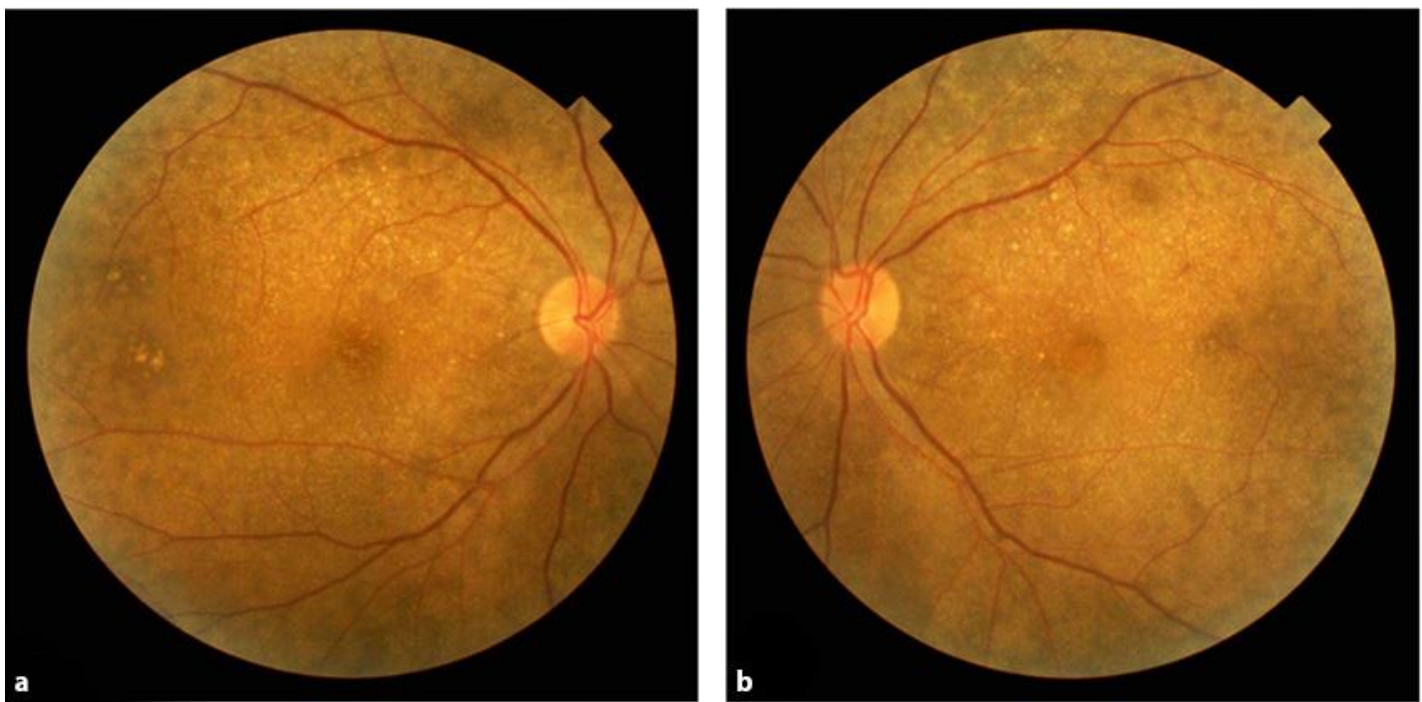

Fig. 1. Fundus photographs of the right eye (a) and the left eye (b) of a patient complaining of blurred vision but with 20/20 vision in both eyes. Cuticular drusen can be seen as many small yellowish sub-RPE deposits.

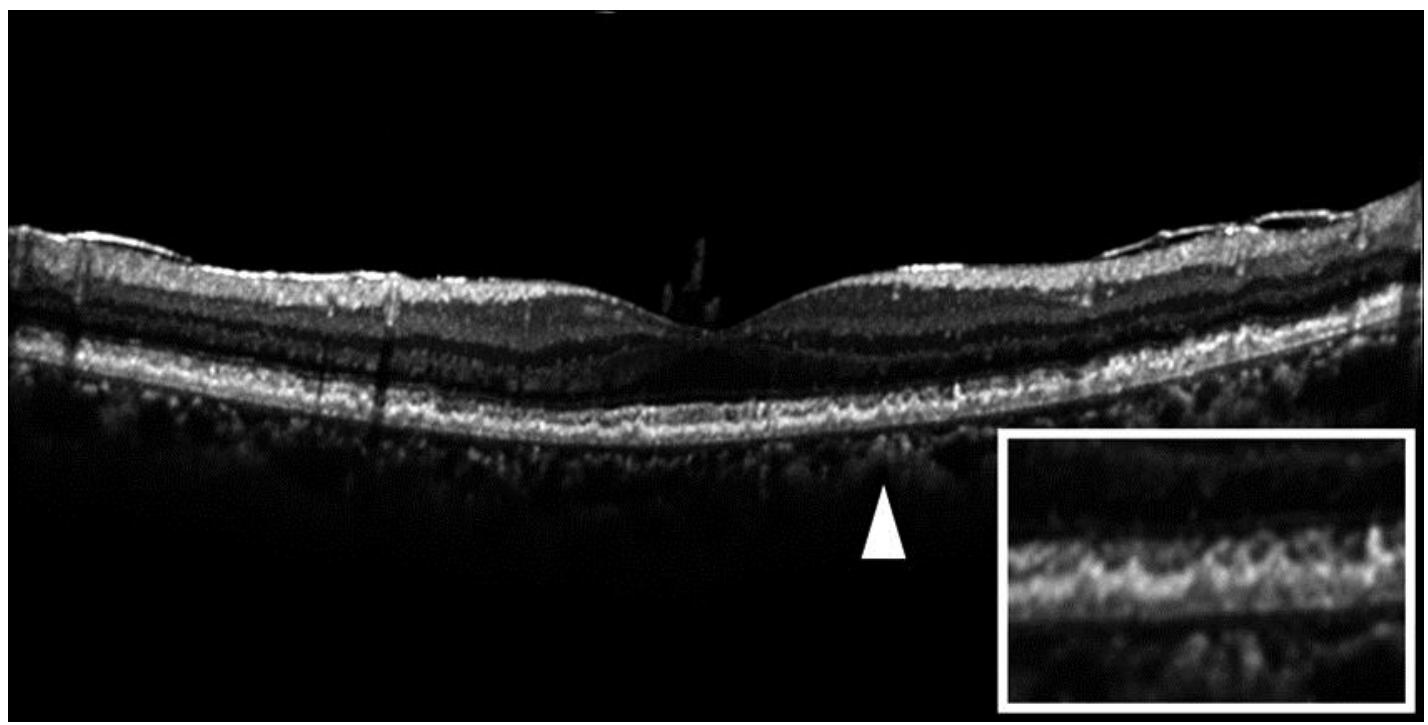

Fig. 2. OCT of the right eye. Cuticular drusen (arrowhead) are seen as small nodular elevations of the RPE with a 'saw-tooth pattern'. 


\section{Case Reports in \\ Ophthalmology}

\begin{tabular}{l|l}
\hline \multicolumn{2}{l}{ Case Rep Ophthalmol 2015;6:127-131 } \\
\hline DOI: 10.1159/000381616 & $\begin{array}{l}\text { ○ 2015 S. Karger AG, Basel } \\
\text { www.karger.com/cop }\end{array}$ \\
\hline
\end{tabular}

Sato et al.: Retinal Angiomatous Proliferation in an Eye with Cuticular Drusen
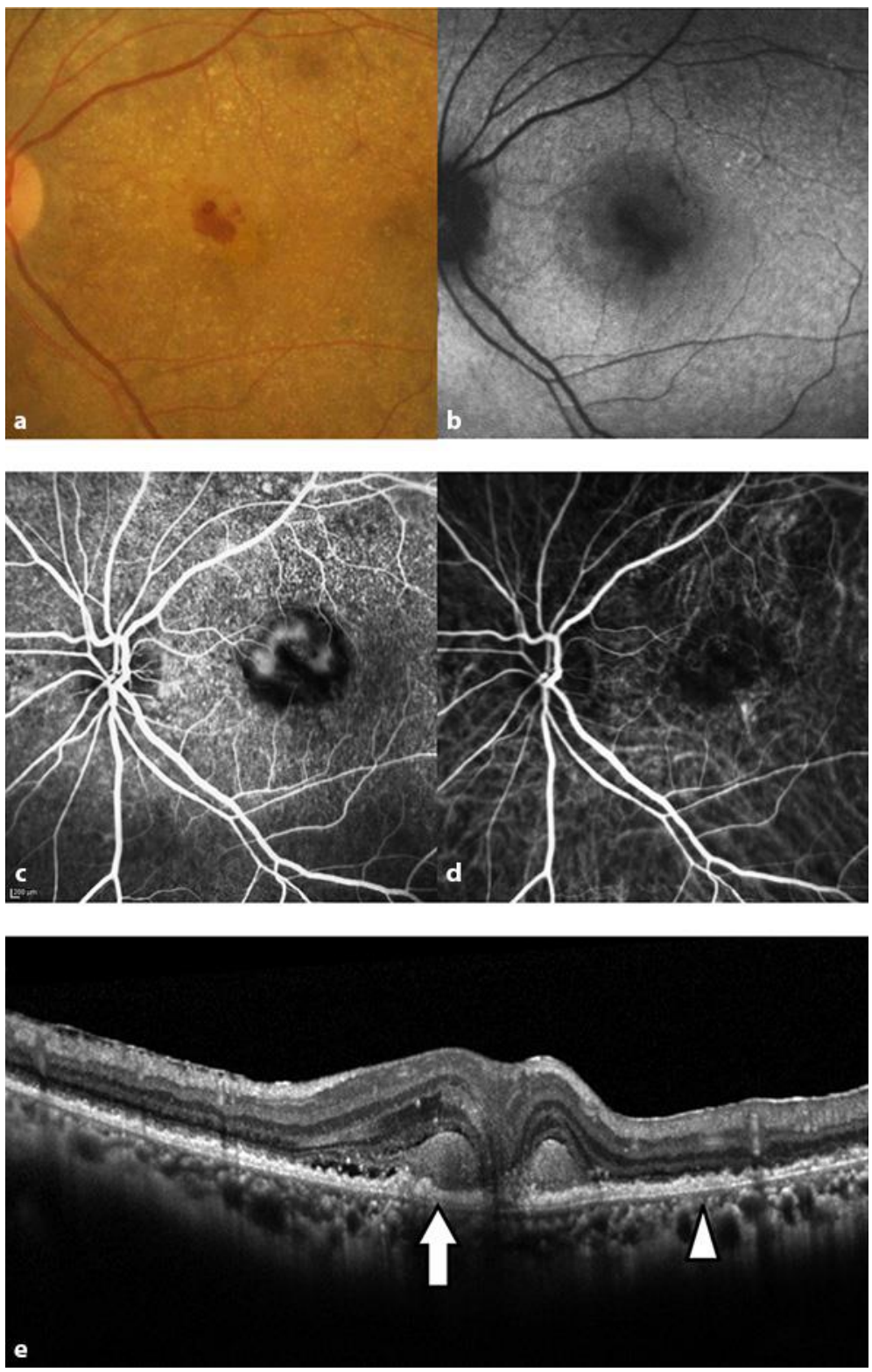

Fig. 3. Fundus photograph (a), autofluorescence image (b), fluorescein and indocyanine green angiograms (c) and OCT image (d) of the left eye. a A fundus photograph shows cuticular drusen and subretinal hemorrhage. b An autofluorescence image shows mottled hyper- and hypofluorescent regions. c, d Fluorescein and indocyanine green angiograms showing a filling of the intraretinal neovascularization. The fluorescein angiogram has a 'stars-in-the-sky' appearance. Fluorescein leakage can be seen around the neovascularization. e OCT shows subretinal exudative changes (arrowhead) and a 'saw-tooth pattern' (arrow). 